\title{
Can Nephrologists Use Ultrasound to Evaluate the Inferior Vena Cava? A Cross-Sectional Study of the Agreement between a Nephrologist and a Cardiologist
}

\author{
José Muniz Pazelia-e Daniel Fagundes Vidigalc Tarcísio Cestari Grossi ${ }^{\mathrm{e}}$ \\ Natália Maria Silva Fernandes ${ }^{a}, b$ Fernando Colugnati ${ }^{a}, b$ \\ Rogério Baumgratz de Paula ${ }^{a, b}$ Hélady Sanders-Pinheiro ${ }^{a, b}$

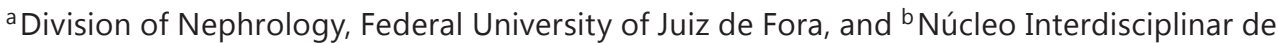 \\ Estudos e Pesquisas em Nefrologia (NIEPEN), Juiz de Fora, ' Clinical Department, Faculty of \\ Medicine of Barbacena (FAME), and dPró-Renal Center of Renal Diseases and Dialysis Unit, \\ Barbacena, Brazil; ' World Interactive Network Focused on Critical Ultrasound (WINFOCUS), \\ Milan, Italy
}

Key Words

Agreement · Fluid status · Hemodialysis · Inferior vena cava · Point-of-care ultrasonography

\begin{abstract}
Background/Aims: The costs and the need for a specialist impair the implementation of ultrasonography for evaluating the inferior vena cava (IVC) to assess the volemic status in hemodialysis patients. We investigated whether a nephrologist with limited ultrasound training can accurately assess the IVC in patients undergoing hemodialysis. Methods: A cardiologist and a nephrologist consecutively measured the indexed IVC expiratory diameter (VCDi) and the IVC collapsibility index (IVCCI) of 52 patients during hemodialysis sessions. In protocol I, the nephrologist used a regular ultrasound system (RUS) and the cardiologist used a cardiovascular ultrasound equipment; in protocol II, the machines were interchanged. Pearson and kappa coefficients and the interexaminer agreement by the Bland-Altman method were calculated. Results: The VCDi measurements showed a strong correlation in both protocols $(r=$ 0.88 and 0.84 in protocols I and II, respectively). The volemic classifications were excellent in protocol I ( $\mathrm{kappa}=0.82$ and 0.93 by VCDi and IVCCI, respectively) and substantial in protocol II (kappa $=0.77$ and 0.75 by VCDi and IVCCI, respectively). The interexaminer agreement on the VCDi measurements was very good in both protocols. Conclusions: Ultrasound evaluation of the IVC can be performed by nephrologists using an RUS to assess the volemic status in hemodialysis patients.


Muniz Pazeli et al.: Can Nephrologists Use Ultrasound to Evaluate the IVC?

A Cross-Sectional Study of the Agreement between a Nephrologist and a Cardiologist

\section{Introduction}

Despite the magnitude of the resources used for treating end-stage renal disease, patients continue to experience high mortality and morbidity rates and reduced quality of life [1]. Inadequate fluid removal during hemodialysis is a critical factor in these patient outcomes. Chronic hypervolemia leads to hypertension, left ventricular hypertrophy, heart failure, and increased mortality and hospitalization rates [2]. On the other hand, hypovolemia is associated with hypotension, decreased quality of life, interruptions of dialysis sessions with reduced solute removal, vascular access thrombosis and loss of residual renal function [3-6].

Although considerable progress has been achieved in the evaluation of dialysis adequacy in relation to the removal of solutes, standardized methods for evaluating the adequacy of blood volume status are currently lacking [7]. Evaluation of the patient's volemic status is difficult with the traditional clinical tools. Clinically estimated dry weight is widely used but is poorly predictive of the volemic status [8]. Currently, other instruments for volemic status evaluation have been developed and validated. Despite the lack of gold standards, related to limitations in accuracy and feasibility, fluid volume has been assessed by using various tools, including ultrasonographic evaluation of the inferior vena cava (IVC-US) [8].

Many authors have reported the potential of IVC-US in patients undergoing dialysis as a better alternative to the clinical estimation of dry weight [9-12]. The estimation of dry weight based on the inferior vena cava (IVC) expiratory diameter (VCDi) and its decrease during inspiration considerably reduces the incidence of events during hemodialysis, reduces left ventricular mass and improves the quality of life $[13,14]$. However, the cost of ultrasound machines and the need for a specialist to operate this equipment impair the widespread implementation of the method [15].

Recently, ultrasound equipment has become more compact, less expensive and with better quality imaging, which has enabled the increasing use of point-of-care ultrasonography [16]. Accordingly, recent studies have demonstrated acceptable accuracy in limited examinations performed by nonspecialists $[17,18]$.

In this study, we sought to determine whether a nephrologist with limited ultrasound training can appropriately assess the IVC in patients undergoing hemodialysis by using a regular ultrasound system (RUS) compared with a cardiologist using a full cardiovascular ultrasound system (CVUS). This is the first study on the accuracy of an RUS for evaluating the VCDi and its inspiratory collapse in patients undergoing hemodialysis.

\section{Materials and Methods}

\section{Study Design}

The IVC-US agreement between a nephrologist without formal ultrasound training and an experienced cardiologist as well as between an RUS and a CVUS were examined by using a cross-sectional design (fig. 1). Our institution ethics review board approved the study and all patients provided written informed consent.

\section{Sample, Setting and Data Collection}

This study was conducted between March and June 2012 at a dialysis outpatient clinic with 168 patients. A convenience sample of 52 patients undergoing chronic hemodialysis was selected. The patients were enrolled consecutively without considering their body habitus or medical history, except for age, i.e. the patients had to be more than 18 years old. The patients were examined first by one researcher and then by the other during dialysis sessions, with a minimum time gap between the examinations (max. $15 \mathrm{~min}$ ). Some patients were examined 
Muniz Pazeli et al: Can Nephrologists Use Ultrasound to Evaluate the IVC?

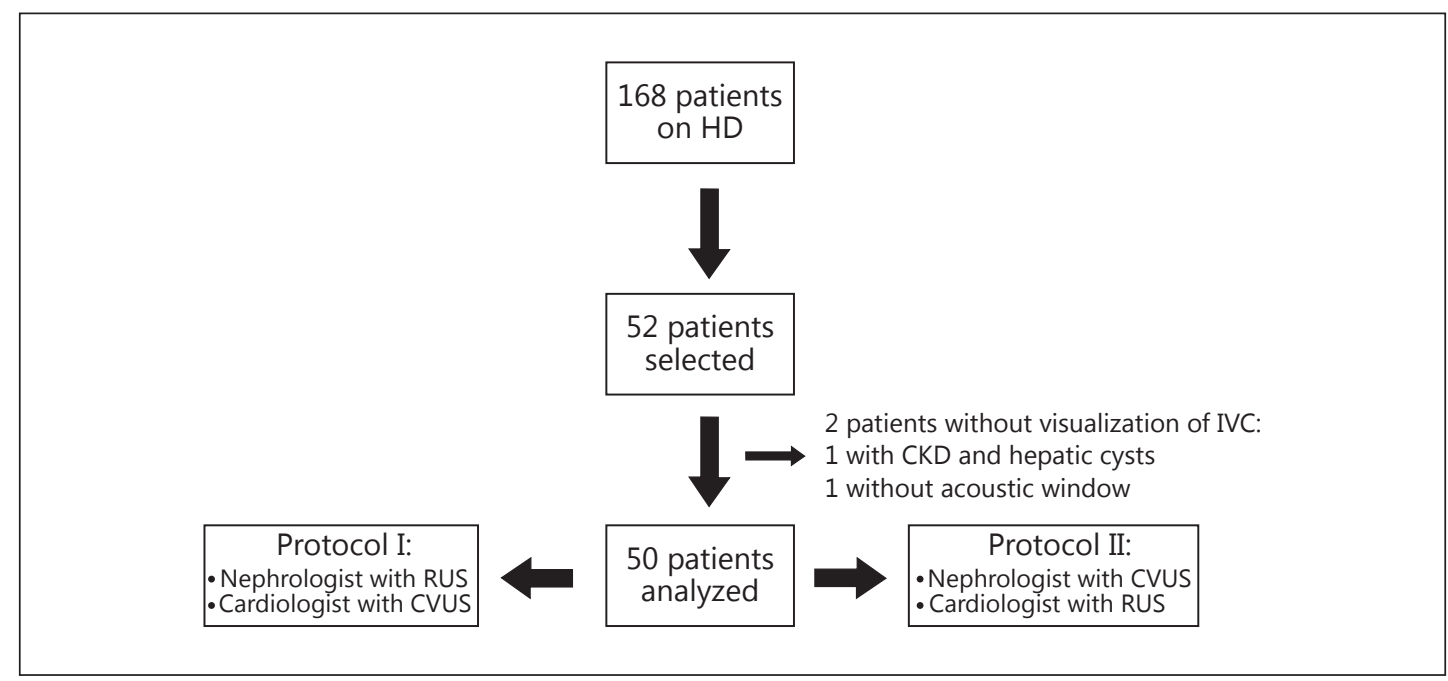

Fig. 1. Design of the study. $\mathrm{HD}=$ Hemodialysis; $\mathrm{CKD}=$ chronic kidney disease.

at the beginning, some in the middle, and some at the end of the dialysis sessions. Using this approach, we planned to evaluate patients in different volemic statuses.

Patient demographic and clinical data that could influence IVC-US, such as age, gender, weight, height, body mass index, etiology of chronic kidney disease and dialysis duration, were collected from the medical files.

\section{Ultrasound Procedures and Volemic Classifications}

A CVUS (t3000CV, Terason, Burlington, Mass., USA) and an RUS (SonoAce Pico, Samsung Medison, Seoul, Korea) were used. The patients underwent a brief echographic examination during their dialysis sessions, in the supine position with $30^{\circ}$ inclination. The probe was placed in the subxiphoid region and a sagittal view of the IVC was obtained by B-mode ultrasonography, just below the diaphragm in the hepatic segment [12]. After visualizing the IVC, a loop was acquired while taking care to maximize the IVC diameter throughout the respiratory cycle, by adjusting the angle between the transducer and the body. Images were frozen and scrolled to find the maximal IVC diameter $\left(\mathrm{IVC}_{\max }\right)$ during passive expiration, immediately after the hepatic vein or within $2.5 \mathrm{~cm}$ of the junction of the IVC with the right atrium, when the hepatic vein could not be visualized. Then, the minimum IVC diameter $\left(\mathrm{IVC}_{\min }\right)$ was measured during passive inspiration. A short, quick inspiratory effort or a 'sniff' by the patients was not allowed, because differences in the magnitude of the inspiratory effort can influence IVC collapse [19].

The indexed VCDi was calculated by dividing the $\mathrm{IVC}_{\max }(\mathrm{in} \mathrm{mm}$ ) by the body surface area (in $\mathrm{m}^{2}$ ). The IVC collapsibility index (IVCCI; in \%) was calculated by using the following standard formula: $\left[\left(\mathrm{IVC}_{\max }-\mathrm{IVC}_{\min }\right) / \mathrm{IVC}_{\max }\right] \times 100$. Volemic status was classified as: hypervolemia, IVCCI $<40 \%$ and/or VCDi $>11.5 \mathrm{~mm} / \mathrm{m}^{2}$, and hypovolemia, IVCCI $>75 \%$ and/or VCDi $<8 \mathrm{~mm} / \mathrm{m}^{2}$. Values between these extremes were considered to indicate normovolemia [9].

\section{Protocols}

In protocol I, 26 patients were examined by the nephrologist, using the RUS. Thereafter, the same patients were examined by the cardiologist using the CVUS. In protocol II, the machines were interchanged between the examiners and the remaining 26 patients were examined consecutively. The examiners were blinded to each other and to the patients' 


\begin{tabular}{l|l}
\hline Nephron Extra 2014;4:82-88 \\
\hline DOI: $10.1159 / 000362170$ & $\begin{array}{l}\text { C 2014 S. Karger AG, Basel } \\
\text { www.karger.com/nne }\end{array}$ \\
\hline Muniz Pazeli et al.: Can Nephrologists Use Ultrasound to Evaluate the IVC? \\
A Cross-Sectional Study of the Agreement between a Nephrologist and a Cardiologist \\
\hline \\
\hline Male gender \\
Age, years \\
Body mass index \\
$<18.5$ & $26(50 \%)$ \\
$18.6-24.9$ & $23.8 \pm 4.8$ \\
$\quad 25-29.9$ & $3(5 \%)$ \\
$>30$ & $36(69 \%)$ \\
Duration of dialysis, months & $9(18 \%)$ \\
Chronic kidney disease etiology & $4(8 \%)$ \\
Hypertensive nephrosclerosis & $54(6-191)$ \\
Diabetic nephropathy & $17(32.7 \%)$ \\
Chronic glomerulonephritis & $12(23 \%)$ \\
Undetermined & $11(21 \%)$ \\
Other & $7(13.5 \%)$ \\
\hline
\end{tabular}

Table 1. Demographic and clinical characteristics of patients (total $n=52$ )
Data represent means \pm standard deviations, medians with ranges in parentheses or numbers with percentages in parentheses.

Table 2. Average values of VCDi and IVCCI on protocols I and II

\begin{tabular}{|c|c|c|c|c|}
\hline & \multicolumn{2}{|l|}{ Protocol I } & \multicolumn{2}{|l|}{ Protocol II } \\
\hline & $\begin{array}{l}\text { nephrologist with } \\
\text { RUS }\end{array}$ & $\begin{array}{l}\text { cardiologist with } \\
\text { CVUS }\end{array}$ & $\begin{array}{l}\text { nephrologist with } \\
\text { CVUS }\end{array}$ & $\begin{array}{l}\text { cardiologist with } \\
\text { RUS }\end{array}$ \\
\hline $\mathrm{VCDi}, \mathrm{mm} / \mathrm{m}^{2}$ & $9.84 \pm 3.61$ & $10.63 \pm 3.63$ & $8.33 \pm 2.29$ & $7.53 \pm 2.29$ \\
\hline IVCCI, \% & $38.0(5.15-100.0)$ & $40.0(5.53-100.0)$ & $50.5(15.0-100.0)$ & $46.4(21.0-100.0)$ \\
\hline
\end{tabular}

Data represent means \pm standard deviations or medians with ranges in parentheses.

clinical status. The examinations were performed within a maximum time gap of $15 \mathrm{~min}$, to avoid significant variations in volemic status (fig. 1).

The nephrologist had limited echocardiographic exposure and no formal training in ultrasonography. He received 30 min of didactic exposure on IVC-US and performed 6 supervised measurements of the IVC.

\section{Statistical Procedures}

Descriptive data are expressed as means \pm standard deviations, medians (ranges) or numbers (percentages). The Shapiro-Wilk test was conducted to assess normality. Pearson and kappa intraclass correlation coefficients were used to evaluate the interobserver correlation of the VCDi measurements and volemic classifications (by the VCDi and IVCCI), respectively, in both protocols. The Bland-Altman method was used for graphical representation of the interexaminer agreement on the VCDi measurements. Statistical analyses were performed by using Stata 11 (StataCorp, College Station, Tex., USA).

\section{Results}

The baseline patient demographic and clinical data are presented in table 1 . Their mean age was $52.8 \pm 15$ years, $50 \%$ of the patients were male and $26 \%$ were classified as overweight or obese. Of the 52 selected patients, 2 were excluded from the analysis because of the 
Table 3. Interobserver kappa correlation of the volemic classifications in the two protocols

\begin{tabular}{llll}
\hline Classification & Correlation, \% & kappa & $\mathrm{p}$ \\
\hline By the VCDi & & & \\
Protocol I & 88.0 & 0.82 & $<0.001$ \\
Protocol II & 88.0 & 0.77 & $<0.001$ \\
By the IVCCI & & & \\
Protocol I & 96.0 & 0.93 & $<0.001$ \\
Protocol II & 84.0 & 0.75 & $<0.001$ \\
\hline
\end{tabular}

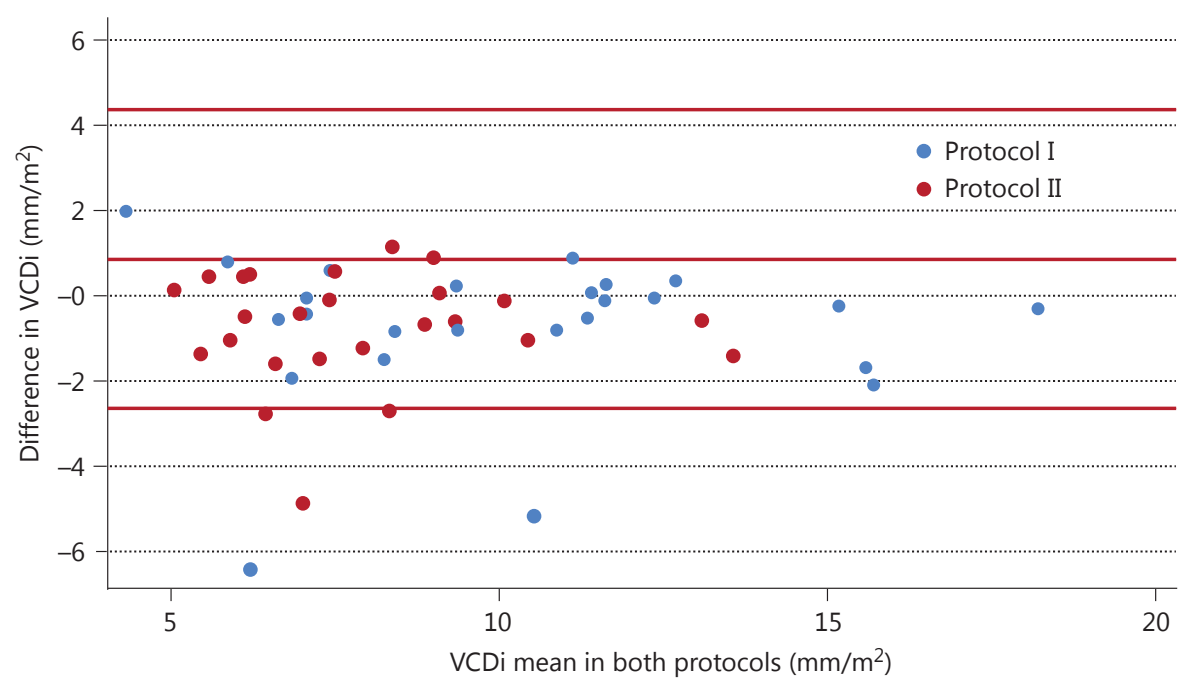

Fig. 2. The Bland-Altman graph.

inability of both examiners to obtain adequate images; 1 patient had polycystic kidney disease and several cysts in the liver, which prevented IVC visualization, and IVC images were impossible to obtain from the other patient. As 50 patients were included in the analysis, 100 images of the IVC ( 50 of the expiratory diameter and 50 of the inspiratory diameter) were obtained by each examiner. In table 2 , we show the average values of VCDi and IVCCI in both protocols.

The VCDi measurements showed a strong correlation in both protocols $(r=0.88, p<$ 0.001 , for both protocols). In the case of the volemic classifications, the kappa values were excellent in protocol I ( 0.82 and 0.93 by the VCDi and IVCCI, respectively). In protocol II, the correlation was lower but substantial ( 0.77 and 0.75 by the VCDi and IVCCI, respectively) (table 3). The Bland-Altman plot showed that the VCDi measurements were close to the central axis, indicating good interexaminer agreement in both protocols (fig. 2).

\section{Discussion}

By this study, we show, for the first time, a strong correlation and agreement between a nephrologist and a cardiologist on IVC-US in patients undergoing hemodialysis. The nephrologist achieved a similar performance even when the equipment was changed. Volemic classification by IVC-US in patients undergoing hemodialysis has been performed by several 
investigators with good accuracy [11-13]. However, in those studies, the examinations were performed by an experienced echocardiographist using a CVUS. In a study which better approaches to ours, Brennan et al. [15] employed medical residents with minimal echocardiographic exposure and no formal training in ultrasonography, but they did not evaluate the agreement with a specialist, which we actually did in our study. In the present study, we compared the results of IVC-US obtained by a nephrologist without formal ultrasound training with those obtained by an experienced echocardiographer, using either an RUS or a CVUS.

The rate of high-quality image acquisition (96\%) in our study was similar to that previously reported (94-95\%) [15, 20,21]. Regarding the factors that traditionally affect the acquisition of good IVC images by ultrasound, we must consider the low frequency of obese patients in our sample.

The Pearson correlation between the nephrologist and the cardiologist for the VCDi measurements in the two protocols was impressive. The study design was innovative and has never been used in the context of IVC-US, because previous studies assessed the reliability between observers by varying only the extent of IVC-US training $[22,23]$. In the case of the kappa correlation, the volemic classification by VCDi was excellent in protocol I and substantial in protocol II. A potential reason for the better correlation in protocol I is that both examiners used equipment they often employ in their daily practice. Fields et al. [22], in an analogous study, observed a similar correlation among bedside clinician sonographers by using a CVUS after extensive training in emergency ultrasonography compared with an experienced sonographer. Randazzo [23] described only fair agreement in a study comparing examinations performed by emergency physician sonographers with limited training in echocardiography using formal echocardiograms; however, the time interval between assessments was longer.

The Pearson correlation coefficient may not be very useful to assess agreement, because it assumes a linear relationship between variables [24]. With regard to the volemic classifications, we should emphasize that they are based on an intervallic range, so even small millimetric differences in the measurements may change the classification. Such a small error size is very common in an operator-dependent method as ultrasonography. In order to settle all these questions, we plotted the VCDi means in a Bland-Altman graph to discard systematic differences between the examiners. Actually, the graph showed good agreement, although one examiner systematically obtained slightly smaller measurements. These differences should have no clinical relevance, because they did not change the volemic classifications, as seen by the high kappa values in both protocols, suggesting that an RUS can be used for evaluating the IVC, unlike the CVUS used in other studies [15, 22]. To the best of our knowledge, the agreement between a cardiologist and a nonspecialist, without formal ultrasound training, has never been studied.

The main limitation of IVC-US is that the method is user dependent. One can obtain a very good image of the IVC, but if the angle between the transducer and the patient's skin is incorrect or if the image is not frozen exactly on time, volemia may be misinterpreted [11]. This limitation is intrinsic to the ultrasound method.

In conclusion, IVC-US can be performed by nephrologists with limited echocardiographic experience using an ordinary ultrasound machine. These findings make IVC-US a potentially useful, noninvasive and repeatable modality for dry weight estimation in dialysis clinics.

\section{Acknowledgements}

José Muniz Pazeli Jr. was granted by Coordenação de Aperfeiçoamento de Pessoal de Nível Superior (CAPES) and by Fundação Instituto Mineiro de Estudos e Pesquisas em Nefrologia (IMEPEN). 


\title{
Disclosure Statement
}

\author{
The authors declare that no competing interests exist.
}

\section{References}

1 Agarwal R: Hypervolemia is associated with increased mortality among hemodialysis patients. Hypertension 2010;56:512-517.

2 Pecoits-Filho R, Bucharles S, Barberato SH: Diastolic heart failure in dialysis patients: mechanisms, diagnostic approach, and treatment. Semin Dial 2012;25:35-41.

-3 London GM: Ultrafiltration intensification for achievement of dry weight and hypertension control is not always the therapeutic gold standard. J Nephrol 2011;24:395-397.

-4 Curatola G, Bolignano D, Rastelli S, Caridi G, Tripepi R, Tripepi G, et al: Ultrafiltration intensification in hemodialysis patients improves hypertension but increases AV fistula complications and cardiovascular events. J Nephrol 2011;24:465-473.

5 Wang AY, Lai K-N: The importance of residual renal function in dialysis patients. Kidney Int 2006;69:17261732.

-6 Parker T III, Hakim R, Nissenson AR, Steinman T, Glassock RJ: Dialysis at a crossroads: 50 years later. Clin J Am Soc Nephrol 2011;6:457-461.

7 McGee S, Abernethy WB III, Simel DL: The rational clinical examination. Is this patient hypovolemic? JAMA 1999;281:1022-1029.

8 Ishibe S, Peixoto AJ: Methods of assessment of volume status and intercompartmental fluid shifts in hemodialysis patients: implications in clinical practice. Semin Dial 2004;17:37-43.

-9 Cheriex EC, Leunissen KM, Janssen JH, Mooy JM, van Hooff JP: Echography of the inferior vena cava is a simple and reliable tool for estimation of 'dry weight' in haemodialysis patients. Nephrol Dial Transplant 1989;4: 563-568.

10 Leunissen KM, Kouw P, Kooman JP, Cheriex EC, de Vries PM, Donker AJ, et al: New techniques to determine fluid status in hemodialyzed patients. Kidney Int Suppl 1993;41:S50-S56.

11 Kusaba T, Yamaguchi K, Oda H, Harada T: Echography of inferior vena cava for estimating fluid removed from patients undergoing hemodialysis. Nihon Jinzo Gakkai Shi 1994;36:914-920.

12 Tetsuka T, Ando Y, Ono S, Asano Y: Change in inferior vena caval diameter detected by ultrasonography during and after hemodialysis. ASAIO J 1995;41:105-110.

13 Chang S-T, Chen C-C, Chen C-L, Cheng H-W, Chung C-M, Yang T-Y: Changes of the cardiac architectures and functions for chronic hemodialysis patients with dry weight determined by echocardiography. Blood Purif 2004;22:351-319.

$\checkmark 14$ Chang ST, Chen CL, Chen CC, Hung KC: Clinical events occurrence and the changes of quality of life in chronic haemodialysis patients with dry weight determined by echocardiographic method. Int J Clin Pract 2004;58: 1101-1107.

15 Brennan JM, Ronan A, Goonewardena S, Blair JEA, Hammes M, Shah D, et al: Handcarried ultrasound measurement of the inferior vena cava for assessment of intravascular volume status in the outpatient hemodialysis clinic. Clin J Am Soc Nephrol 2006;1:749-753.

16 Moore CL, Copel JA: Point-of-care ultrasonography. N Engl J Med 2011;364:749-757.

17 Ferrada P, Anand RJ, Whelan J, Aboutanos MA, Duane T, Malhotra A, et al: Qualitative assessment of the inferior vena cava: useful tool for the evaluation of fluid status in critically ill patients. Am Surg 2012;78:468-470.

18 Stawicki SP, Braslow BM, Panebianco NL, Kirkpatrick JN, Gracias VH, Hayden GE, et al: Intensivist use of handcarried ultrasonography to measure IVC collapsibility in estimating intravascular volume status: correlations with CVP. J Am Coll Surg 2009;209:55-61.

19 Mintz GS, Kotler MN, Parry WR, Iskandrian AS, Kane SA: Real-time inferior vena caval ultrasonography: normal and abnormal findings and its use in assessing right-heart function. Circulation 1981;64:1018-1025.

20 Moreno FL, Hagan AD, Holmen JR, Pryor TA, Strickland RD, Castle CH: Evaluation of size and dynamics of the inferior vena cava as an index of right-sided cardiac function. Am J Cardiol 1984;53:579-585.

21 Bendjelid KK, Romand J-AJ, Walder BB, Suter PMP, Fournier GG: Correlation between measured inferior vena cava diameter and right atrial pressure depends on the echocardiographic method used in patients who are mechanically ventilated. J Am Soc Echocardiogr 2002;15:944-949.

22 Fields JM, Lee PA, Jenq KY, Mark DG, Panebianco NL, Dean AJ: The interrater reliability of inferior vena cava ultrasound by bedside clinician sonographers in emergency department patients. Acad Emerg Med 20;18: 98-101.

23 Randazzo MR: Accuracy of emergency physician assessment of left ventricular ejection fraction and central venous pressure using echocardiography. Acad Emerg Med 2003;10:973-977.

24 van Stralen KJ, Jager KJ, Zoccali C, Dekker FW: Agreement between methods. Kidney Int 2008;74:1116-1120. 